\title{
Interval Between Neoadjuvant Chemoradiation and Surgery for the Management of Rectal Cancer
}

\author{
Sergio Huerta
}

Received: 1 January 2011 / Accepted: 26 March 2011 /Published online: 12 April 2011

(C) 2011 The Society for Surgery of the Alimentary Tract

\section{Dear Editor:}

In the manuscript "Neoadjuvant Therapy for Rectal Cancer: The Impact of Longer Interval Between Chemoradiation and Surgery", Dr. de Campos-Lobato ${ }^{1}$ continues to add evidence to the positive oncological effects of achieving a pathological complete response (pCR). ${ }^{2}$ Additionally, their manuscript provides alternative treatment strategies in terms of timing in the trimodality (surgery, chemotherapy, and radiotherapy) approach for the management of rectal cancer. While the rate of complications was similar in both of the groups analyzed, the cohort that had a waiting interval of $\geq 8$ weeks demonstrated a higher rate of $\mathrm{pCR}$. This approach is novel and continues to add tools in the armamentarium in the management of patients affected with rectal cancer.

There are, however, a few issues that remain at large: (1) how can we identify the patients likely to response to neoadjuvant modalities? Our experience shows that up to $50 \%$ of patients show a partial response; while, over $20 \%$ do not respond to this strategy and, in fact, the tumor continues to grow. ${ }^{3}$ Thus, waiting longer clearly benefits the segment of the patients that achieve a pCR, but how can we identify this cohort patients preoperatively? (2) The 8 -week period mark stems from the group's previous work, ${ }^{4}$

\section{S. Huerta $(\bowtie)$}

University of Texas Southwestern/Dallas VA Medical Center, 4500 S. Lancaster Road, Surgical Services (112),

Dallas, TX 75216, USA

e-mail: Sergio.huerta@utsouthwestern.edu in which a longer interval was an independent predictor for pCR. However, can we wait even longer (10 to 12 weeks)? Will a longer interval even result in a superior rate of pCR? What should the limit be? Thus, we need randomized trials to assess the best interval for surgical intervention following neoadjuvant chemoradiation. Preclinical studies might also suggest a time interval for the best tumor response. (3) Should chemotherapy be continued during the interval period? We should exercise caution in widening the interval prior to surgical intervention as some patients might experience tumor growth during treatment. These patients should at least undergo restaging strategies at a given interval between the waiting period of neoadjuvant chemoradiation and surgical intervention. This interval is also best addressed in the context of a randomized control trial.

\section{References}

1. de Campos-Lobato LF, Geisler DP, da Luz MA et al. (2010) Neoadjuvant Therapy for Rectal Cancer: The Impact of Longer Interval Between Chemoradiation and Surgery. J Gastrointest Surg 15(3):444-50

2. Maas M, Nelemans PJ, Valentini V et al. Long-term outcome in patients with a pathological complete response after chemoradiation for rectal cancer: a pooled analysis of individual patient data. Lancet Oncol 2010; 11:835-844.

3. Huerta S, Hrom J, Gao XH et al. Tissue microarray constructs to predict a response to chemoradiation in rectal cancer. Digestive and Liver Disease 2010; 42:679-684.

4. Kalady MF, de Campos-Lobato LF, Stocchi L et al. (2011) Predictive factors of pathologic complete response after neoadjuvant chemoradiation for rectal cancer. Ann Surg (in press) 